\title{
Simian adenoviruses as vaccine vectors
}

\author{
Susan J Morris ${ }^{*, 1}$, Sarah Sebastian', Alexandra J Spencer ${ }^{1} \&$ Sarah C Gilbert
}

\begin{abstract}
Replication incompetent human adenovirus serotype 5 (HAdV-C5) has been extensively used as a delivery vehicle for gene therapy proteins and infectious disease antigens. These vectors infect replicating and nonreplicating cells, have a broad tissue tropism, elicit high immune responses and are easily purified to high titers. However, the utility of HAdV-C5 vectors as potential vaccines is limited due to pre-existing immunity within the human population that significantly reduces the immunogenicity of $\mathrm{HAdV}-\mathrm{C} 5$ vaccines. In recent years, adenovirus vaccine development has focused on simian-derived adenoviral vectors, which have the desirable vector characteristics of HAdV-C5 but with negligible seroprevalence in the human population. Here, we discuss recent advances in simian adenovirus vaccine vector development and evaluate current research specifically focusing on clinical trial data.
\end{abstract}

First draft submitted: 22 June 2016; Accepted for publication: 19 August 2016;

Published online: 15 September 2016

Replication incompetent human adenovirus serotype 5 (HAdV-C5) has been extensively used as a delivery vehicle for gene therapy proteins and infectious disease antigens. However, these vaccine vectors are limited within the clinical setting due to the high seroprevalence, $40-45 \%$ in the USA and up to $90 \%$ in residents of sub-Saharan Africa, to HAdV-C5 within the human population [1-3]. A recent Phase IIb trial (STEP trial) of an HAdV-C5 vaccine expressing antigens of HIV-1 was abruptly halted due to lack of efficacy [4] and the subsequent finding that participants in one subgroup with circulating neutralizing antibodies to HAdV-C5 prior to vaccination showed a nonsignificant increase in acquiring HIV infection $[5,6]$. In light of these studies there has been a growing interest in generating vaccines from rare human adenovirus serotypes and nonhuman adenovirus serotypes, which have negligible seroprevalence in the human population [7,8]. Leading nonhuman adenovirus candidates include vectors derived from simian adenoviruses (SAds) [9-11] and in particular those derived from chimpanzee adenoviruses (termed ChAds or AdCs) [12-14]. Although SAds are closely related to human adenoviruses [15] the hypervariable regions of the main immunogen, hexon, are significantly different from HAdV-C5 that they circumvent preexisting immunity to HAdV-C5. Replication incompetent SAd vaccine vectors lack the essential growth viral transactivator genes encoded by the $\mathrm{E} 1$ region and thus vector production requires the expression of E1 proteins in trans. SAds and HAdV-C5 share a close homology in the E1 region allowing simian E1 deleted adenovirus vector complementation in cell lines originally derived for complementation of HAdV-C5 E1 deleted vectors [16]. An added benefit is that there is no risk of generating replication competent adenoviruses through recombination events between the SAd genome and the complementing region within the host cell as the E1 flanking regions are different between HAdV-C5 and SAds [12,14].

'Jenner Institute, ORCRB, University of Oxford, Off Roosevelt Drive, Headington, Oxford, OX3 7DQ, UK

*Author for correspondence: susan.morris@ndm.ox.ac.uk

\section{KEYWORDS}

- BAC recombineering

- chimpanzee adenoviruses

- clinical trials $\bullet$ simian

adenoviruses $\bullet$ vector

vaccine 
Vaccine vectors derived from ChAd3, 7, 6, virus [22], Ebola [23], SARS [7], hepatitis C [24,25], $9,32,33,63$ and 68 have been generated [12] and tested in preclinical settings for immunogenicity toward a wide range of pathogens including malaria [17-19], HIV [20,21], influenza rabies virus [26] and Rift Valley fever [27]. These vectors have been demonstrated to induce immune responses at very low doses in mice $\left(1-3 \times 10^{6}\right.$ viral particles). Protective immunity

(A)

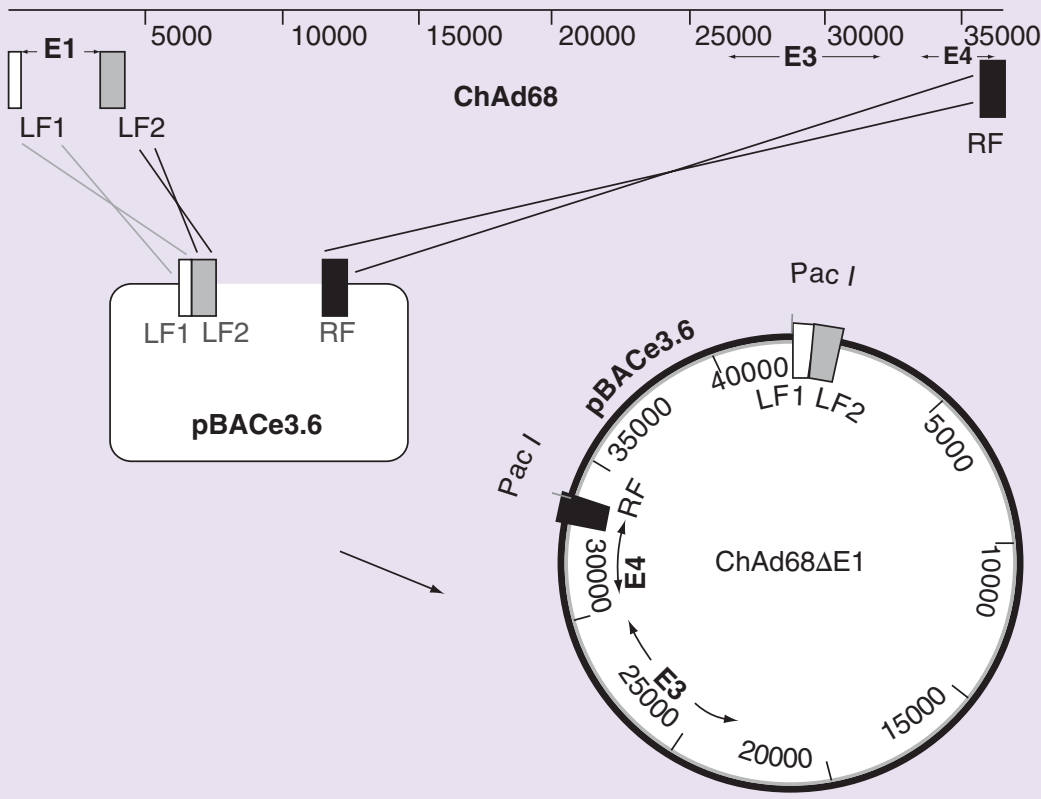

(B)
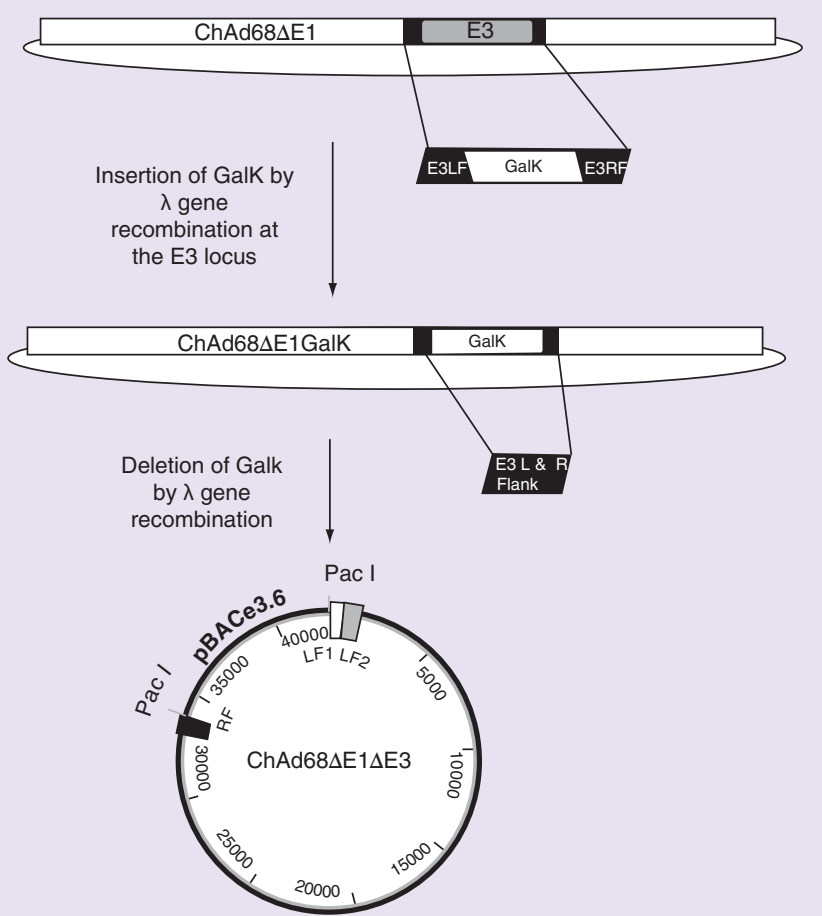

(C)
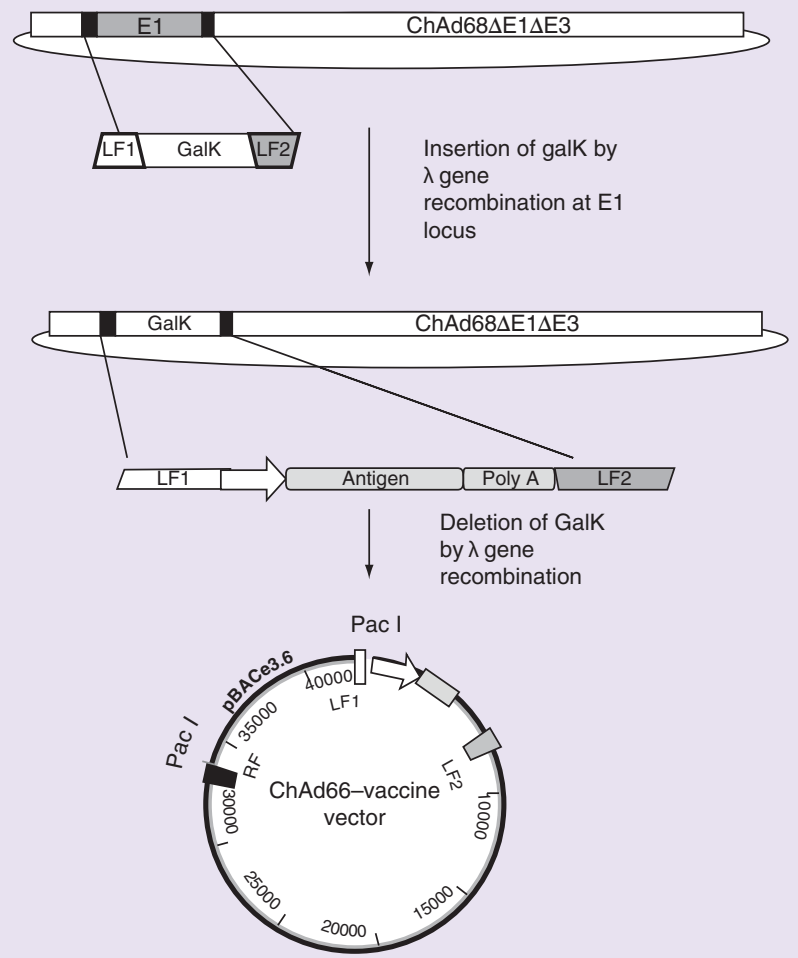
Figure 1. Generation of a molecular clone of ChAd68 (see facing page). (A) Insertion of ChAd68 genomic DNA into the PBAC 'rescue vector' by gap repair. The E1 LF1 and 2 and terminal right hand side region (RF) are amplified from ChAd68 genomic DNA and cloned into pBACe3.6 to produce a $B A C$ adenovirus rescue clone. Recombination occurs between LF1 and LF2 of the isolated ChAd68 genome and the BAC rescue clone and the RF of ChAd68 genome and the BAC rescue clone. The resulting product is a $\mathrm{BAC}$ containing an $\mathrm{E} 1$ deleted $\mathrm{ChAd} 68$ genome. (B) Excision of the $\mathrm{E} 3$ region of ChAd68 by recombineering. First, the galactokinase gene (GalK) is amplified from pGalK using primers containing sequences homologous to the flanking region of E3 (E3LF and E3RF). The E3 region is replaced by the GalK gene using $\lambda$ red recombination. The GalK gene is subsequently replaced by a PCR product consisting of E3LF and E3RF, again using $\lambda$ red recombination. The resulting product is a BAC containing an E1E3-deleted ChAd68 genome. (C) Insertion of an antigen cassette at the E1 locus. First, the GalK gene is amplified from pGalK using primers containing sequences homologous to the flanking region of E1 (LF1 and LF2). The E1 region is replaced by the GalK gene using $\lambda$ red recombination. The GalK gene is subsequently replaced by a PCR product consisting of LF1-antigen-expression cassette-LF2 using $\lambda$ red recombination. The resulting product is a BAC containing an E1E3-deleted ChAd68 genome with an antigen-expression cassette at the E1 locus.

BAC: Bacterial artificial chromosome; ChAd68: Chimpanzee adenovirus 68; LF: Left flanking region; RF: Right flanking region.

in preclinical models was equal to, or greater than, that induced by equivalent HAdV-C5 vectors for ChAd63-derived vaccine vectors [12]. Promising preclinical data have led to the use of SAd vaccine vectors in clinical trials where they have been shown to have a good safety and immunological profile [12].

\section{- SAd vaccine vector design \& development}

Key considerations in the design of SAd vectors for use as vaccines are similar to those for HAdV-C5. The vaccine vector must be nonreplicating and unlike adenovirus gene therapy vectors have negligible immune modulatory activity. Hence, SAd vectors lack the E1 region encoding viral transactivator proteins which are essential for virus growth and the $\mathrm{E} 3$ region encoding immunomodulatory proteins.

The advent of bacterial artificial chromosomes (BACs) coupled to bacteriophage $\lambda$ red recombination (recombineering) technology has facilitated the manipulation of large virus genomes [28]. Using this approach, linear DNA adenovirus genomes isolated from nonhuman primates have been cloned for use as viral vectors. The first stage, following virus isolation and genome sequencing, is either the amplification or artificial synthesis of: two products homologous to the left arm of the genome which flank the E1 region, and; one product, approximately $1000 \mathrm{bp}$, homologous to the right arm of the genome each incorporating a unique restriction enzyme site for cloning and genome excision for vector production. These fragments are assembled and inserted into a BAC by conventional restriction enzyme cloning. The virus genome is then inserted into the BAC clone by single-step gap repair homologous recombination to generate an E1 deleted viral vector molecular clone (Figure 1A). The recombineering system is then used to allow seamless deletion of the adenovirus E3 immunomodulatory genes. Firstly, the bacterial galactokinase gene ( $G a l K)$ is amplified from the plasmid, pGalK, such that it contains approximately 50 bp homology arms flanking the $\mathrm{E} 3$ region, this gene is inserted at the E3 locus of the BAC-rescued adenovirus genome by $\lambda$ red recombination. Clones are screened for growth on galactose as this phenotype is attributed to the GalK gene product. The GalK gene is then removed by $\lambda$ red recombination with a PCR product comprised of the E3 left and right flanking region only (Figure 1B). Positive clones are selected on 2-deoxygalactose media, which prevents growth of bacteria expressing the GalK gene. Further manipulation using $\lambda$ red recombination firstly to insert the GalK gene and then to exchange it for an antigen-expression cassette at the E1 locus completes the engineering of the vaccine vector (Figure 1C) [29]. The linear virus genome is excised from the BAC using unique restriction enzymes, usually PacI or PmeI, and transfected into complementing cells to generate the viral vector. The antigen cassette typically consists of a strong promoter such as the minimal cytomegalovirus (CMV) immediate early promoter, to drive antigen expression, the antigen of interest 
and a polyadenylation signal. We have generated a molecular toolbox that allows the insertion of any gene easily into a set region within the ChAd genome by inserting universal cassettes expressing a bacteria antibiotic resistance gene flanked by specific recombination sequences, such as att $\mathrm{R} 1$ and att $\mathrm{R} 2$, derived from bacteriophage $\lambda$ (note this system is based on the gateway cloning system from Invitrogen), into our ChAd derived vaccine vectors at the E1 locus and/or the E3 locus. Shuttle plasmids containing an antigen-expression cassette flanked by specific recombination sites paired with those present in the genome (e.g., attR1/R2 recombination sequence requires att $\mathrm{L} 1 / \mathrm{L} 2$ recombination sequence) allow site-specific recombination in the presence of an enzyme mixture containing bacteriophage $\lambda$ integrase, integration host factor and excisionase (Figure 2).

Although the deleted E1 region from SAds is complemented by HAdV-C5 E1 proteins constitutively expressed by human embryonic kidney (HEK293) cells or PerC.6 cells, viral yields vary depending on SAd serotype. High yields of Pan5, ChAd68 (also referred to as Pan 6 or sAd25) and Pan7, all derived from chimpanzees can be obtained from HEK293 cells [16], whereas ChAd1 yields are poor [30]. For virus vectors with poor replication, further genome manipulation has been shown to increase yields. In the case of $\mathrm{HAdV}-\mathrm{C} 5$, the $\mathrm{E} 4$ gene products in particular those from orf3, orf4, orf6 and orf6/7 coordinate their function with the E1 proteins (E1A and E1B 55K) and host cell cofactors to bind, regulate and derepress several cellular functions during viral multiplication [31-36]. Manipulation of the E4 region can therefore be a promising means of increasing virus yields. We have recently described the generation of a chimeric vaccine vector, ChAdOxl, derived from ChAd serotype $\mathrm{Y} 25$ engineered by $\lambda$ red recombination to exchange the native E4 orf4 orf6 and orf6/7 genes for those from HAdV-C5. This vector showed an increase in hexon protein production from HEK293 cells compared with the ChAd parent virus [37]. Using this approach we have also recently generated $\mathrm{ChAdOx} 2$, an E1/E3-deleted vaccine vector derived from ChAd68 with a modified E4 region to increase virus yields in HEK293 cells (Figure 3). Tatsis et al. report that exchanging the left and right inverted terminal repeats and packaging

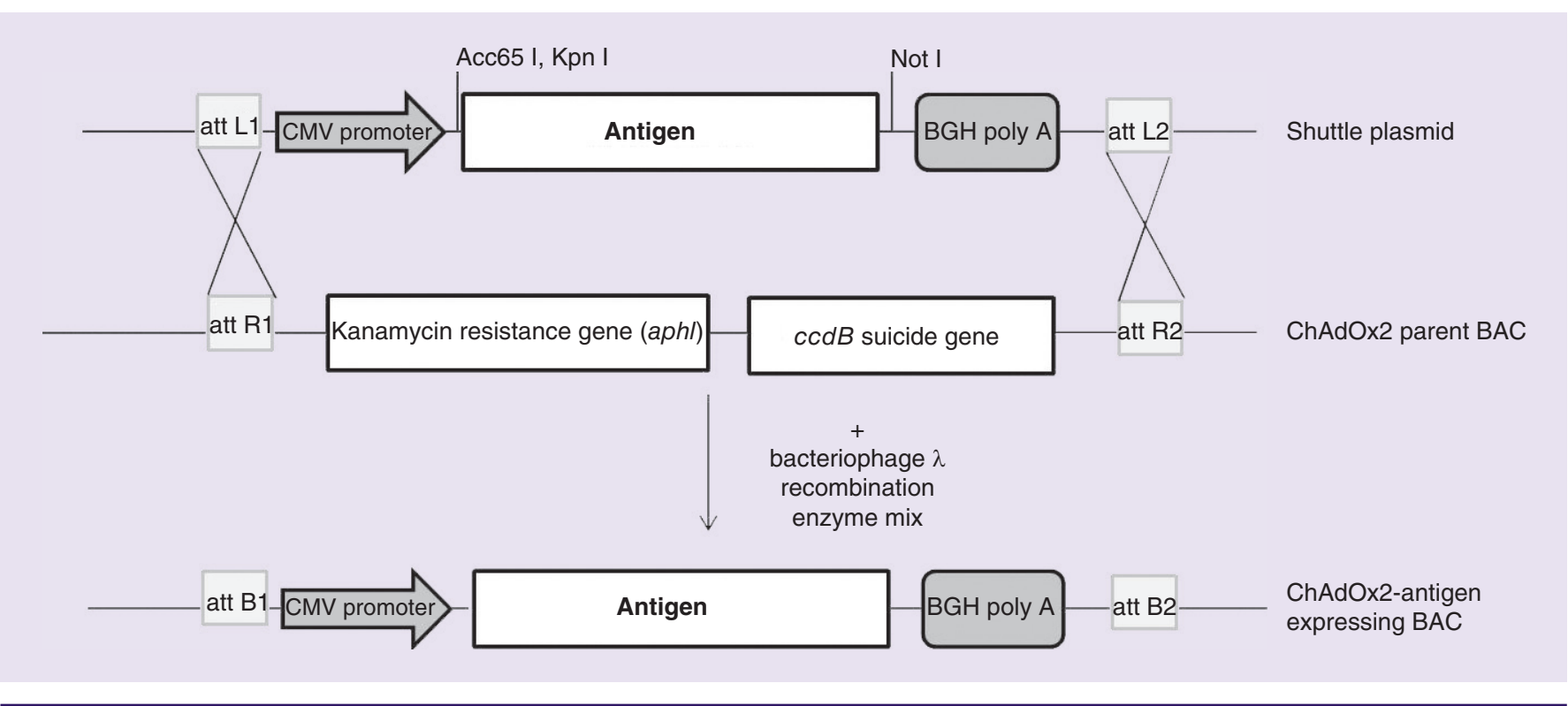

Figure 2. Insertion of an antigen-expression cassette into adenovirus vector using att recombination sites. A universal cassette expressing a bacteria antibiotic resistance gene and $\mathrm{ccdB}$ suicide gene flanked by the specific recombination sequences, attR1 and attR2 is located at the E1 locus and/or the E3 locus of the BAC-adenovirus genome clone. Shuttle plasmids containing an antigenexpression cassette flanked by specific recombination sites paired with those present in the adenovirus genome (attL1/L2) allow site-specific recombination in the presence of an enzyme mixture containing bacteriophage $\lambda$ integrase, integration host factor and excisionase.

BAC: Bacterial artificial chromosome; ChAdOx2: E1/E3 deleted adenovirus vector derived from ChAd68 with a modified E4 region; CMV: Cytomegalovirus. 
signal allowed the complementation of ChAd1 viral vectors [30]. Therefore further modification of SAds, which have previously been neglected as candidates for viral vaccines due to poor productivity in HEK293 cells, could allow complementation and thus expand the ever growing list of SAd viral vaccines.

\section{- SAd vector engineering to improve immunogenicity}

Adenovirus vaccine vectors, regardless of parental origin, can induce humoral, mucosal and cellular immune responses, depending on the route of administration. However, although the Tand $\mathrm{B}$-cell responses elicited are good for most vectors, the level of immunological potency can differ depending on adenovirus vector parental strain/serotype $[12,38]$. For example, when the two simian vectors ChAdOx1 (derived from Y25) and ChAdOx2 (derived from ChAd68), which both carried a green fluorescent protein (GFP) expression cassette in the E1 locus, were compared, the T-cell response elicited to GFP by IFN- $\gamma$ ELISpot assay, antigen-specific production of IFN- $\gamma$ in response to stimulation with the immunodominant GFP peptide was significantly higher in ChAdOx2 mice compared with ChAdOx1 vaccinees (Figure 4). Similar to human adenovirus $\mathrm{C}$ serotypes, most $\mathrm{SAd}$ s use the coxsackie adenovirus receptor, which is present in heart tissue, brain tissue and epithelial and endothelial cells, as the host cell receptor. However, ChAd1 is closely related to human adenoviruses of subgroup B2 and uses CD46, a regulatory protein in the complement system, which is expressed on all human cells except erythrocytes, as the host cell receptor [30]. ChAd1, therefore, has a broad infectivity tropism, allowing it to utilize cells and infection pathways that are not accessible to other Ad serotypes. ChAd7 (sAdV24)-based vectors induce superior protective mucosal immunity in the respiratory tract, particularly following mucosal immunization. This enhanced mucosal immunogenicity could be related to prolonged persistence of the ChAd7 vectors in antigenpresenting cells [39]. Furthermore, differences between vaccine vector immune responses between hosts have also been reported. In mice, after intramuscular immunization, HAdV-C5 based vectors elicited cellular and humoral adaptive responses of higher magnitudes compared with ChAdOx1 and ChAd68 whereas in cattle, cellular and humoral immune responses were

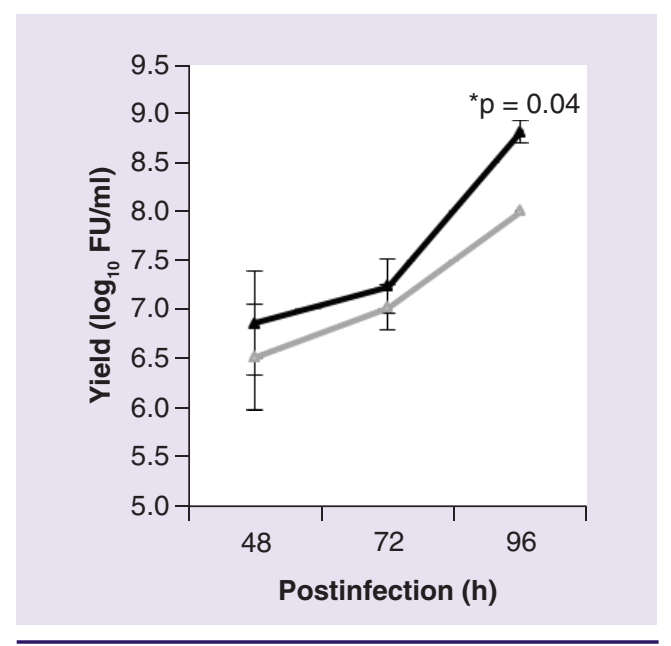

Figure 3. Growth of ChAdOx2 compared with ChAd68. E1-complementing human embryonic kidney HEK293 cells were infected with one multiple of infection of viral vectors ChAdOx2 (black line) or ChAd68 (gray line) each expressing GFP from the E1 locus. Samples were taken at 48 and $96 \mathrm{~h}$ postinfection. Virus yield was determined by titration in triplicate on HEK293 cells and GFP-positive cells counted 48-h postinfection. Results are expressed as $\log _{10}$ fluorescent units per milliliter from two separate experiments with triplicate titrations for each sample. Student's unpaired $t$-test was used to statistically analyze the results and the mean with standard deviation is depicted.

FU: Fluorescent unit; GFP: Green fluorescent protein.

at least equivalent, if not higher, in magnitude after ChAdOx1 vaccination compared with HAdV-C5 [40].

Many current studies are driven by a desire to improve SAd vaccine immunogenicity and have focused on producing novel antigen presentation on the viral vaccine surface through engineering of the virus capsid proteins. The SAd hexon protein is the major capsid protein with each viral particle containing 240 copies of the hexon trimer. Antigen epitopes introduced in the hypervariable regions of the hexon protein will be displayed on the virus surface. Zhou et al. showed that a linear epitope to the influenza virus M2 protein located within hypervariable region 1 , but not hypervariable region 4, of ChAd68 hexon induced a higher antibody response than when $\mathrm{M} 2$ was expressed as a transgene at the E1 locus [3]. Introduction of an epitope from coxsackievirus A16 into hypervariable region 1 and an epitope from 


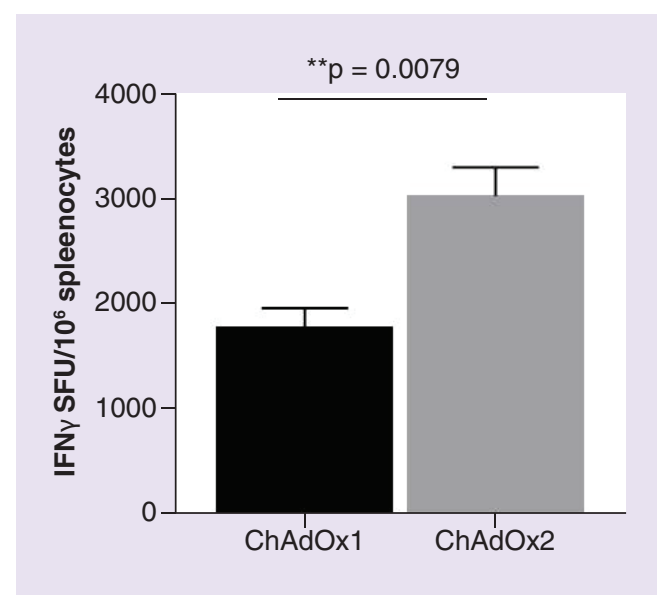

Figure 4. Immunogenicity of ChAdOx1-eGFP compared with ChAdOx2-eGFP. Female BALB/c mice (four per group) were injected intramuscularly with $10^{8}$ infectious units of vector and spleens harvested 2 weeks later to measure the response to GFP by IFN- $\gamma$ ELISPOT. Results are expressed as SFUs per million splenocytes. Mann-Whitney test was used to statistically analyze the results and the mean with SEM is depicted.

GFP: Green fluorescent protein; SFU: Spotforming unit.

enterovirus 71 into hypervariable region 2 of ChAd68 generated a bivalent vaccine which elicited a high immune response for hand, foot and mouth disease [41]. The SAd fiber protein has also been a target for improving vaccine immunogenicity. The trimeric fiber protein protrudes from the penton base at each of the 12 vertices of the capsid. The fiber proteins are responsible for host cell receptor binding and thus viral transduction of cells. Engineering of HAdV-C5 fiber has shown that viral vaccine cell tropism can be altered and that insertion of antigen epitopes can elicit an immune response. Fiber modifications of SAds have also been tested. Insertion of the tripeptide arg-gly-asp (RGD) motif into the fiber of ChAd7 expressing an antigen for
Pseudomonas aeruginosa enhanced mucosal protective immunogenicity by increasing the level of infection of cells expressing high levels of $\alpha v \beta 3$ and $\alpha v \beta 5$ integrins, such as dendritic cells [42]. Taken together these data show the importance of choosing the correct vector serotype for the desired host and location of immune response, in conjunction with modifications to tropism and method of antigen presentation to ensure the generation of an optimal adenovirus vector vaccine.

\section{- Clinical trials}

Of the many SAds isolated, vectorized and tested in preclinical studies, four have been advanced into clinical vaccine trials to date (Table 1). The first to do so, ChAd63-METRAP, a ChAd63 vector encoding the malaria antigen thrombospondin-related adhesion protein (TRAP) fused to a multiepitope (ME) string containing epitopes from several malaria antigens, was initially used in a Phase I dose and route finding study to assess safety and immunogenicity [43]. In addition to being tested on its own, it was also evaluated as a priming agent in a prime-boost regimen with the modified vaccinia Ankara (MVA) poxviral vector expressing METRAP. In this trial, doses between $1 \times 10^{8}$ and $2 \times 10^{11} \mathrm{vp}$ of ChAd63 were found to be safe and elicited high levels of antigen-specific T cells, especially when part of a prime-boost schedule. Based on these encouraging results, the ChAd63 vector (encoding a variety of malaria antigens) has since been used in 21 further Phase I and II malaria vaccine trials, mostly in combination with an MVA boost (reviewed in [44]) but also on its own [45-48], with a protein-in-adjuvant boost [49] or in combination with an MVA vector and the virus like particle vaccine RTS,S (licensed under the name Mosquirix ${ }^{\mathrm{TM}}$ ), which expresses the Asn-Ala-Asn-Pro (NANP) repeat and T-cell epitope sequences from Plasmodium falciparum circumsporozoite protein [50,51]. In all

\section{Table 1. Simian adenoviral vectors used in clinical trials.}

\begin{tabular}{|c|c|c|c|c|}
\hline $\begin{array}{l}\text { Vector (species isolated } \\
\text { from) }\end{array}$ & Classification (group) & Trial (phase) & Pathogen/disease & Ref. \\
\hline PanAd3 (Pan paniscus) & C & I & RSV & {$[53,54]$} \\
\hline ChAd3 (Pan troglodytes) & C & $\mathrm{I}, \mathrm{II}$ & Ebola, HCV & {$[25,55-58]$} \\
\hline ChAd63 (Pan troglodytes) & $E$ & I, II & Malaria, HIV & {$[43,52]$} \\
\hline $\begin{array}{l}\text { ChAdOx1 (modified from } \\
\text { Pan troglodytes Y25) }\end{array}$ & $E$ & 1 & $\begin{array}{l}\text { Influenza A, prostate } \\
\text { cancer, tuberculosis }\end{array}$ & {$[53,59-60]$} \\
\hline
\end{tabular}


of these trials, the ChAd63 vector has consistently proved to be an excellent priming agent for a strong $\mathrm{CD}^{+}{ }^{+} \mathrm{T}$-cell response. In addition to malaria vaccine trials, ChAd63 has also been used in clinical studies of HIV vaccines [52]. Here, ChAd63 encoding the HIVconsv immunogen derived from the functionally most conserved regions of the HIV-1 proteosome was tested in combination with MVA or plasmid DNA vaccination, and the prime-boost vaccination regimens were able to induce high frequencies of $\mathrm{CD}^{+} \mathrm{T}$ cells specific for the conserved regions of HIV-1. Taking together all clinical trials, the ChAd63 vector has been assessed in more than 1000 individuals to date, including infants and children, and the observed high immunogenicity for both T-cell and antibody responses warrants further development of this vector.

Of equal interest, another chimpanzee adenoviral vector has also made significant progress in a total of ten clinical trials to date: ChAd3 was first used in $\mathrm{HCV}$ vaccine trials in heterologous prime-boost schedules together with HAdV-6 [25] or MVA [55]. Both trials recorded durable and broad T-cell responses to the HCV antigen. ChAd3 has also been evaluated in the context of the recent Ebolavirus outbreak. Starting in late 2014, ChAd3 encoding the Ebolavirus glycoprotein (ChAd3-EBO-Z developed by GSK) was fast-tracked into four Phase I trials in the UK, USA, Mali and Uganda [56-57,61], as well as a Phase I/II trial in Switzerland [62]. At doses between $10^{10}$ and $10^{11} \mathrm{vp}$, the vaccine showed an acceptable safety profile and significant humoral immunogenicity up to 6 months postvaccination. In fact, antibody-responses 4 weeks after a single dose of ChAd3-EBO-Z were equivalent to those seen in the much publicized Phase III clinical trial of vesicular stomatitis virus-based Ebola vaccine, which showed $100 \%$ efficacy [63]. These trials therefore suggest that a single dose of the adenoviral vector may be enough to confer protective efficacy in a ring-vaccination scenario. A large Phase II safety/efficacy study in Liberia was also planned for 2015/16 [58,64], but due to a decline in the incidence of Ebola by the start of the study, efficacy outcomes will likely not be assessable.

The third chimpanzee vector to be tested in the clinic, ChAdOx1, was developed at Oxford University, and is based on the chimpanzee Y25 adenovirus [37]. A Phase I trial of ChAdOx1 encoding the conserved influenza antigens nucleoprotein and matrix protein 1 (ChAdOx1
$\mathrm{NP}+\mathrm{M} 1)$ found high levels of antigen-specific $T$ cells, which were comparable to those elicited previously in trials using the ChAd63 vector encoding malaria antigens [59]. Two further trials involving ChAdOx1 are currently underway, as vaccine candidates against tuberculosis and prostate cancer $[53,60]$.

The most recent simian adenoviral vector to undergo clinical evaluation is PanAd3, which is based on an adenovirus originally isolated from a bonobo [12]. The PanAd3 vector encoding three antigens of the respiratory syncytial virus (RSV) was tested at a dose of $5 \times 10^{10} \mathrm{vp}$ in healthy adults, with either intramuscular or intranasal administration, followed by a booster vaccination of MVA encoding the same antigens [54,65]. This prime boost regimen was shown to induce robust RSV-specific T-cell responses post-boost, independent of the route of priming, although as expected, intranasal vaccination with PanAd3 resulted in lower levels of systemic RSV-specific $T$ cells than intramuscular administration. The acceptable safety profile and immunogenicity observed in this trial warrant further clinical investigation of the PanAd3 vector.

\section{Conclusion \& future perspective}

SAd viral vaccines, especially those derived from ChAds, are a viable alternative to HAdV-C5derived viral vaccines. Promising preclinical data have led to the use of ChAd vaccine vectors in clinical trials for a variety of infectious diseases and have shown good safety and immunological profiles in Phase I trials. In the next few years many of these vectors should enter Phase II and III clinical trials providing us with a better understanding of SAd vector immunogenicity in humans. ChAd vectors are also currently being developed as delivery vehicles for antigens against cancers, such as prostate [66] and breast cancer as well as chronic diseases, for example, Crohn's disease. Thus, the use of SAds as a general vaccine vector should become well established in the coming years.

The employment of certain SAdV-based vectors as vaccines may not be suitable for some populations. Although found less frequently than antibodies to HAdV-C5, neutralizing antibodies to some chimpanzee adenovirus serotypes have been detected in humans from sub-Saharan Africa, Brazil and China [2-3,7-8]. Neutralizing antibodies to the vector significantly reduce the specific immune response against the transgene product and thus can be detrimental to the efficacy of a 
vaccine vector. These data show that an understanding of the immune state of the population to be vaccinated is essential when designing a vaccine vector. Vectors derived from other SAds, rare human adenovirus serotypes and adenoviruses from other species are being developed and may provide additional or alternative vaccine vectors.

A wealth of preclinical data relating to the use of SAd viral vectors as vaccines are available; however, different antigens, virus serotypes and immunization strategies have made it difficult to compare vectors head to head. Future studies need to focus on comparisons of vector backbones, antigen presentation and tropism modification so that information can be collated to allow the establishment of guidelines for the generation of optimal SAd vector vaccines for different hosts and immunological outcomes. These data together with advances in molecular engineering strategies and manufacturing technology will open up the possibility of rapidly generating SAd vector vaccines to combat emerging diseases, such as Ebola and Zika.

\section{Acknowledgements}

The authors acknowledge S Chinnakannan and

$M$ Ulaszewska for their assistance with molecular biology

and immunogenicity testing of $C h A d O x 2$, respectively.
Financial \& competing interests disclosure

The generation of ChAdOx2 vector described was funded by a Wellcome Trust Award to Adrian Hill (reference: $095540 / z / 11 / z)$. The authors have ownership of a patent for ChAdOxl and ChAdOx2. S Gilbert is a co-founder of, consultant to and shareholder in Vaccitech plc, which is developing viral vectored vaccines. The authors have no other relevant affliations or financial involvement with any organization or entity with a financial interest in or financial conflict with the subject matter or materials discussed in the manuscript apart from those disclosed.

No writing assistance was utilized in the production of this manuscript.

\section{Ethical conduct of research}

The authors state that they have obtained appropriate institutional review board approval or have followed the principles outlined in the Declaration of Helsinki for all human or animal experimental investigations.

\section{Open access}

This article is distributed under the terms of the Creative Commons Attribution License 4.0 which permits any use, distribution, and reproduction in any medium, provided the original author(s) and the source are credited. To view a copy of the license, visit http://creativecommons. org/licenses/by/4.0/

\section{EXECUTIVE SUMMARY}

Vaccine vectors derived from simian adenoviruses provide a viable alternative to human adenovirus serotype 5 vaccine vectors

- Simian adenovirus (SAd) vaccine vectors circumvent pre-existing human adenovirus serotype 5 (HAdV-C5) immunity.

- Most SAd vaccine vectors can be grown in HAdV-C5 complementing cell lines.

- Protective immunity in preclinical models for a range of antigens was equal to, or greater than, that induced by equivalent HAdV-C5 vectors for ChAd63-derived vaccine vectors.

Recombineering technology allows genetic manipulation of the adenovirus genome to generate a range of vaccines with different phenotypic \& immunogenic properties

- SAd viral vaccines are E1E3-deleted viruses.

- Manipulation of the E4 region or inverted terminal repeats increases yields of viral vectors in HEK293 cells.

- Modification of the fiber and hexon proteins provides novel antigen presentation on the surface of SAd viral vectors.

- SAd viral vector immunological potency can differ depending on adenovirus vector parental strain/serotype.

- Parental SAd strain, antigen presentation, the host to be vaccinated and immune response required, for example, mucosal immunogenicity should all be considered in the design of a SAd viral vector.

ChAd vaccine vectors have been shown to have a good safety \& immunological profile in clinical trials

- ChAd viral vaccines against malaria, Ebola, HIV, influenza, respiratory syncytial virus, HCV, tubercolosis and prostate cancer have all progressed to Phase I clinical trials.

- ChAd viral vectors elicit high levels of antigen-specific T cells and/or neutralizing antibodies when administered as part of a prime boost regimen. 


\section{References}

Papers of special note have been highlighted as:

- of interest; $\bullet$ of considerable interest

1 Nwanegbo E, Vardas E, Gao W et al. Prevalence of neutralizing antibodies to adenoviral serotypes 5 and 35 in the adult populations of The Gambia, South Africa, and the United States. Clin. Diagn. Lab. Immunol. 11(2), 351-357 (2004).

2 Dudareva M, Andrews L, Gilbert SC et al. Prevalence of serum neutralizing antibodies against chimpanzee adenovirus 63 and human adenovirus 5 in Kenyan children, in the context of vaccine vector efficacy. Vaccine 27(27), 3501-3504 (2009).

3 Zhang S, Huang W, Zhou X, Zhao Q, Wang Q, Jia B. Seroprevalence of neutralizing antibodies to human adenoviruses type- 5 and type-26 and chimpanzee adenovirus type-68 in healthy Chinese adults. J. Med. Virol. 85(6), 1077-1084 (2013).

4 Buchbinder SP, Mehrotra DV, Duerr A et al. Efficacy assessment of a cell-mediated immunity HIV-1 vaccine (the Step Study): a double-blind, randomised, placebocontrolled, test-of-concept trial. Lancet 372(9653), 1881-1893 (2008).

5 Catanzaro AT, Koup RA, Roederer M et al. Phase 1 safety and immunogenicity evaluation of a multiclade HIV-1 candidate vaccine delivered by a replication-defective recombinant adenovirus vector. J. Infect. Dis. 194(12), 1638-1649 (2006).

6 Priddy FH, Brown D, Kublin J et al. Safety and immunogenicity of a replicationincompetent adenovirus type $5 \mathrm{HIV}-1$ clade B $\mathrm{gag} / \mathrm{pol} / \mathrm{nef}$ vaccine in healthy adults. Clin. Infect. Dis. 46(11), 1769-1781 (2008).

7 Xiang Z, Li Y, Cun A et al. Chimpanzee adenovirus antibodies in humans, subSaharan Africa. Emerg. Infect. Dis. 12(10), 1596-1599 (2006).

8 Ersching J, Hernandez MI, Cezarotto FS et al. Neutralizing antibodies to human and simian adenoviruses in humans and new-world monkeys. Virology 407(1), 1-6 (2010).

9 Abbink P, Maxfield LF, Ng'ang'a D et al. Construction and evaluation of novel rhesus monkey adenovirus vaccine vectors. J. Virol. 89(3), 1512-1522 (2015).

10 Duncan M, Cranfield MR, Torano $\mathrm{H}$ et al. Adenoviruses isolated from wild gorillas are closely related to human species $\mathrm{C}$ viruses. Virology 444(1-2), 119-123 (2013).

11 Roy S, Medina-Jaszek A, Wilson MJ et al. Creation of a panel of vectors based on ape adenovirus isolates. J. Gene Med. 13(1), 17-25 (2011).
12 Colloca S, Barnes E, Folgori A et al. Vaccine vectors derived from a large collection of simian adenoviruses induce potent cellular immunity across multiple species. Sci. Transl. Med. 4(115), 115ra112 (2012).

13 Tatsis N, Tesema L, Robinson ER et al. Chimpanzee-origin adenovirus vectors as vaccine carriers. Gene Ther. 13(5), 421-429 (2006).

14 Farina SF, Gao GP, Xiang ZQ et al. Replication-defective vector based on a chimpanzee adenovirus. J. Virol. 75(23), 11603-11613 (2001).

15 Roy S, Gao G, Clawson DS, Vandenberghe LH, Farina SF, Wilson JM. Complete nucleotide sequences and genome organization of four chimpanzee adenoviruses. Virology 324(2), 361-372 (2004).

16 Roy S, Gao G, Lu Y et al. Characterization of a family of chimpanzee adenoviruses and development of molecular clones for gene transfer vectors. Hum. Gene Ther. 15(5), 519-530 (2004).

17 Bauza K, Malinauskas T, Pfander C et al. Efficacy of a plasmodium vivax malaria vaccine using ChAd63 and modified vaccinia Ankara expressing thrombospondin-related anonymous protein as assessed with transgenic Plasmodium berghei parasites. Infect. Immun. 82(3), 1277-1286 (2014).

18 Reyes-Sandoval A, Sridhar S, Berthoud T et al. Single-dose immunogenicity and protective efficacy of simian adenoviral vectors against Plasmodium berghei. Eur. J. Immunol. 38(3), 732-741 (2008).

19 Goodman AL, Epp C, Moss D et al. New candidate vaccines against blood-stage Plasmodium falciparum malaria: prime-boost immunization regimens incorporating human and simian adenoviral vectors and poxviral vectors expressing an optimized antigen based on merozoite surface protein 1 . Infect. Immun. 78(11), 4601-4612 (2010).

20 Fitzgerald JC, Gao GP, Reyes-Sandoval A et al. A simian replication-defective adenoviral recombinant vaccine to HIV-1 gag. J. Immunol. 170(3), 1416-1422 (2003).

21 Pinto AR, Fitzgerald JC, Gao GP, Wilson $\mathrm{JM}$, Ertl HC. Induction of $\mathrm{CD}^{+}{ }^{+} \mathrm{T}$ cells to an HIV-1 antigen upon oral immunization of mice with a simian E1-deleted adenoviral vector. Vaccine 22 (5-6), 697-703 (2004).

22 Roy S, Kobinger GP, Lin J et al. Partial protection against $\mathrm{H} 5 \mathrm{~N} 1$ influenza in mice with a single dose of a chimpanzee adenovirus vector expressing nucleoprotein. Vaccine 25 (39-40), 6845-6851 (2007).
23 Kobinger GP, Feldmann H, Zhi Y et al. Chimpanzee adenovirus vaccine protects against Zaire Ebola virus. Virology 346(2), 394-401 (2006).

24 Fattori E, Zampaglione I, Arcuri M et al. Efficient immunization of rhesus macaques with an HCV candidate vaccine by heterologous priming-boosting with novel adenoviral vectors based on different serotypes. Gene Ther. 13(14), 1088-1096 (2006).

25 Barnes E, Folgori A, Capone S et al. Novel adenovirus-based vaccines induce broad and sustained T cell responses to HCV in man. Sci. Transl. Med. 4(115), 115ra111 (2012).

- This describes the first clinical use of a ChAd3-vectored vaccine.

26 Zhou D, Cun A, Li Y, Xiang Z, Ertl HC. A chimpanzee-origin adenovirus vector expressing the rabies virus glycoprotein as an oral vaccine against inhalation infection with rabies virus. Mol. Ther. 14(5), 662-672 (2006).

27 Warimwe GM, Lorenzo G, Lopez-Gil E et al. Immunogenicity and efficacy of a chimpanzee adenovirus-vectored Rift Valley fever vaccine in mice. Virol. J. 10, 349 (2013).

-. Demonstrated immunogenicity and complete protective efficacy of a single dose simian adenovirus-vectored vaccine in the livestock species.

28 Ruzsics Z, Lemnitzer F, Thirion C. Engineering adenovirus genome by bacterial artificial chromosome (BAC) technology. Methods Mol. Biol. (Clifton, NJ) 1089, 143-158 (2014).

29 Warming S, Costantino N, Court DL, Jenkins NA, Copeland NG. Simple and highly efficient BAC recombineering using galK selection. Nucleic Acids Res. 33(4), e36 (2005).

30 Tatsis N, Blejer A, Lasaro MO et al. A CD46-binding chimpanzee adenovirus vector as a vaccine carrier. Mol. Ther. 15(3), 608-617 (2007).

31 Weinberg DH, Ketner G. Adenoviral early region 4 is required for efficient viral DNA replication and for late gene expression. J. Virol. 57(3), 833-838 (1986).

32 Querido E, Blanchette P, Yan Q et al. Degradation of $\mathrm{p} 53$ by adenovirus E4orf6 and E1B55K proteins occurs via a novel mechanism involving a Cullin-containing complex. Genes Dev. 15(23), 3104-3117 (2001).

33 Querido E, Morrison MR, Chu-Pham-Dang $\mathrm{H}$, Thirlwell SW, Boivin D, Branton PE. Identification of three functions of the 
adenovirus e4orf6 protein that mediate $\mathrm{p} 53$ degradation by the E4orf6-E1B55K complex. J. Virol. 75(2), 699-709 (2001).

34 Blanchette P, Kindsmuller K, Groitl P et al. Control of mRNA export by adenovirus E4orf6 and E1B55K proteins during productive infection requires E4orf6 ubiquitin ligase activity. J. Virol. 82(6), 2642-2651 (2008).

35 Woo JL, Berk AJ. Adenovirus ubiquitinprotein ligase stimulates viral late mRNA nuclear export. J. Virol. 81(2), 575-587 (2007).

36 Dobner T, Horikoshi N, Rubenwolf S, Shenk T. Blockage by adenovirus E4orf6 of transcriptional activation by the $\mathrm{p} 53$ tumor suppressor. Science 272(5267), 1470-1473 (1996).

37 Dicks MD, Spencer AJ, Edwards NJ et al. A novel chimpanzee adenovirus vector with low human seroprevalence: improved systems for vector derivation and comparative immunogenicity. PLoS ONE 7(7), e 40385 (2012).

-• Describes the production of a simian adenovirus-based vaccine vector starting from wild-type, replication competent virus.

38 Quinn KM, Da Costa A, Yamamoto A et al. Comparative analysis of the magnitude, quality, phenotype, and protective capacity of simian immunodeficiency virus gag-specific $\mathrm{CD}^{+} \mathrm{T}$ cells following human-, simian-, and chimpanzee-derived recombinant adenoviral vector immunization. J. Immunol. 190(6), 2720-2735 (2013).

39 Krause A, Whu WZ, Xu Y, Joh J, Crystal RG, Worgall S. Protective anti-Pseudomonas aeruginosa humoral and cellular mucosal immunity by AdC7-mediated expression of the $P$. aeruginosa protein $\mathrm{OprF}$. Vaccine 29(11), 2131-2139 (2011).

40 Dicks MD, Guzman E, Spencer AJ et al. The relative magnitude of transgene-specific adaptive immune responses induced by human and chimpanzee adenovirus vectors differs between laboratory animals and a target species. Vaccine 33(9), 1121-1128 (2015).

41 Zhang C, Yang Y, Chi Y et al. Hexonmodified recombinant E1-deleted adenoviral vectors as bivalent vaccine carriers for Coxsackievirus A16 and Enterovirus 71. Vaccine 33(39), 5087-5094 (2015).

42 Krause A, Whu WZ, Qiu J et al. RGD capsid modification enhances mucosal protective immunity of a non-human primate adenovirus vector expressing Pseudomonas aeruginosa OprF. Clin. Exp. Immunol. 173(2), 230-241 (2013).
43 O'Hara GA, Duncan CJ, Ewer KJ et al. Clinical assessment of a recombinant simian adenovirus ChAd63: a potent new vaccine vector. J. Infect. Dis. 205(5), 772-781 (2012).

-. First clinical use of a simian adenovirus vector demonstrating both safety and immunogenicity.

44 Sebastian S, Gilbert SC. Recombinant modified vaccinia virus Ankara-based malaria vaccines. Expert Rev. Vaccines 15(1), 91-103 (2016).

45 De Barra E, Hodgson SH, Ewer KJ et al. A Phase Ia study to assess the safety and immunogenicity of new malaria vaccine candidates ChAd63 CS administered alone and with MVA CS. PLoS ONE 9(12), e115161 (2014).

46 Sheehy SH, Duncan CJ, Elias SC et al. Phase Ia clinical evaluation of the safety and immunogenicity of the Plasmodium falciparum blood-stage antigen AMA1 in ChAd63 and MVA vaccine vectors. PLoS ONE 7(2), e31208 (2012).

47 Biswas S, Choudhary P, Elias SC et al. Assessment of humoral immune responses to blood-stage malaria antigens following ChAd63-MVA immunization, controlled human malaria infection and natural exposure. PLoS ONE 9(9), e107903 (2014).

48 A Phase Ia Clinical Trial to Assess the Safety and Immunogenicity of New Plasmodium Falciparum Malaria Vaccine Candidates ChAd63 RH5 Alone and with MVA RH5. https://clinicaltrials.gov

49 Hodgson SH, Choudhary P, Elias SC et al. Combining viral vectored and protein-inadjuvant vaccines against the blood-stage malaria antigen AMA1: report on a Phase 1a clinical trial. Mol. Ther. 22(12), 2142-2154 (2014).

50 A Safety and Efficacy Study of Concomitant Administration of ChAd63/MVA ME-TRAP + RTS,S. https://clinicaltrials.gov

51 A Safety and Efficacy Study of ChAd63/MVA METRAP + RTS,S. https://clinicaltrials.gov

52 Hayton EJ, Rose A, Ibrahimsa U et al. Safety and tolerability of conserved region vaccines vectored by plasmid DNA, simian adenovirus and modified vaccinia virus ankara administered to human immunodeficiency virus type 1-uninfected adults in a randomized, single-blind Phase I trial. PLoS ONE 9(7), e101591 (2014).

- Employed prime boost combinations involving adenovirus, DNA and MVA vectored vaccines.
53 Safety Study of ChAdOx1 85A Vaccination With and Without MVA85A Boost in Healthy Adults. https://clinicaltrials.gov

54 Green CA, Scarselli E, Sande CJ et al. Chimpanzee adenovirus- and MVA-vectored respiratory syncytial virus vaccine is safe and immunogenic in adults. Sci. Transl. Med. 7(300), 300ra126 (2015).

- Describes the first clinical study of a PanAd3-vectored vaccine.

55 Swadling L, Capone S, Antrobus RD et al. A human vaccine strategy based on chimpanzee adenoviral and MVA vectors that primes, boosts, and sustains functional HCV-specific T cell memory. Sci. Transl. Med. 6(261), 261ra153 (2014).

56 Ledgerwood JE, Dezure AD, Stanley DA et al. Chimpanzee adenovirus vector Ebola vaccine - preliminary report. $N$. Engl. J. Med. doi:10.1056/NEJMoa1410863 (2014) (Epub ahead of print).

57 Rampling T, Ewer K, Bowyer G et al. A monovalent chimpanzee adenovirus ebola vaccine - preliminary report. $N$. Engl. J. Med. doi:10.1056/NEJMoa1411627 (2015) (Epub ahead of print).

58 Kennedy SB, Neaton JD, Lane HC et al. Implementation of an Ebola virus disease vaccine clinical trial during the Ebola epidemic in Liberia: design, procedures, and challenges. Clin Trials doi: $10.1177 / 1740774515621037$ (2016) (Epub ahead of print).

59 Antrobus RD, Coughlan L, Berthoud TK et al. Clinical assessment of a novel recombinant simian adenovirus $\mathrm{ChAdOx} 1$ as a vectored vaccine expressing conserved Influenza A antigens. Mol. Ther. 22(3), 668-674 (2014).

- Describes the first clinical use of a ChAdOx1 vectored vaccine.

60 VAccination in Prostate caNCEr (VANCE). https://clinicaltrials.gov

61 Tapia MD, Sow SO, Lyke KE et al. Use of ChAd3-EBO-Z Ebola virus vaccine in Malian and US adults, and boosting of Malian adults with MVA-BN-Filo: a Phase 1, single-blind, randomised trial, a Phase $1 \mathrm{~b}$, open-label and double-blind, dose-escalation trial, and a nested, randomised, double-blind, placebocontrolled trial. Lancet Infect. Dis. 16(1), 31-42 (2016).

62 De Santis O, Audran R, Pothin E et al. Safety and immunogenicity of a chimpanzee adenovirus-vectored Ebola vaccine in healthy adults: a randomised, double-blind, placebo-controlled, dose-finding, Phase $1 / 2$ a study. Lancet Infect. Dis. doi:10.1016/ 
S1473-3099(15)00486-00487 (2015) (Epub ahead of print).

63 Ewer K, Rampling T, Venkatraman N et al. A monovalent chimpanzee adenovirus Ebola vaccine boosted with MVA. N. Engl. J. Med. 374(17), 1635-1646 (2016).

64 Partnership for Research on Ebola Vaccines in Liberia (PREVAIL).

https://clinicaltrials.gov
65 Green CA, Scarselli E, Voysey M et al. Safety and immunogenicity of novel respiratory syncytial virus (RSV) vaccines based on the RSV viral proteins F, N and M2-1 encoded by simian adenovirus (PanAd3-RSV) and MVA (MVA-RSV); protocol for an open-label, dose-escalation, single-centre, Phase 1 clinical trial in healthy adults. BMJ Open 5(10), e008748 (2015).
66 Cappuccini F, Stribbling S, Pollock E, Hill $\mathrm{AV}$, Redchenko I. Immunogenicity and efficacy of the novel cancer vaccine based on simian adenovirus and MVA vectors alone and in combination with PD-1 mAb in a mouse model of prostate cancer. Cancer Immunol. Immunother. doi:10.1007/ s00262-016-1831-1838 (2016) (Epub ahead of print). 